\title{
Outlier-robust estimation of uncertain-input systems with applications to nonparametric FIR and Hammerstein models
}

\author{
Riccardo Sven Risuleo and Håkan Hjalmarsson
}

\begin{abstract}
In this paper, we present an extension of the class of uncertain-input models to handle cases of measurements with outliers. The general uncertain-input model framework allows us to treat system identification problems in which a linear system, represented by its impulse response, is subject to an input about which we have partial information. Both the impulse response and the input are modeled as Gaussian processes and the kernels are used to encode the information available. The whole model is then estimated using an approximate empirical Bayes approach. We extend the uncertain-input model framework to non-Gaussian measurement models by considering the noise precisions as realizations of a Gamma prior. We validate the approach on a dataset of linear systems and on a dataset of Hammerstein systems where the measurements are corrupted by outliers.
\end{abstract}

Index Terms-Identification, Machine Learning, Uncertain systems

\section{INTRODUCTION}

$\mathbf{I}$ $\mathrm{N}$ the classical system identification framework, the independent variable exciting the system is usually thought of as a decision variable that is perfectly known. However, in many applications, the input signal may be noisy or unknown. For instance, the Hammerstein cascade in Figure 1 may be seen as a linear system (represented by the vector of impulse response samples $g$ ) subject to the unknown input $w$. In this case, we may have some partial information about the input $w$ because we may know something about the function $f(\cdot)$ that generated it (e.g., it that is smooth or increasing, or that it is a saturation).

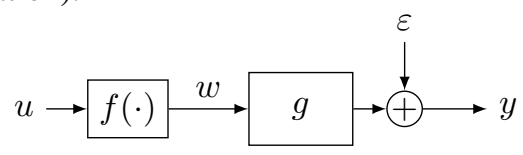

Fig. 1. A Hammerstein cascade.

In the uncertain-input (UI) framework (proposed in [1]), the prior information available about the input and the system is encoded into the mean functions and the covariance matrices of Gaussian process models [2], using a kernel-based approach [3], [4]:

$$
w \sim \mathcal{N}\left(w ; \mu_{w}, \Sigma_{w}\right), \quad g \sim \mathcal{N}\left(g ; \mu_{g}, \Sigma_{g}\right) .
$$

This work was supported by the Swedish Research Council through the project System identification: Unleashing the algorithms, contract 2015-05285, and the research environment NewLEADS - New Directions in Learning Dynamical Systems, contract 2016-06079.

R. S. Risuleo and Håkan Hjalmarsson are with the Department of Automatic control, School of Electrical Engineering and Computer Science, KTH Royal Institute of Technology, Stockholm, Sweden risuleo@kth.se, hjalmars@kth.se

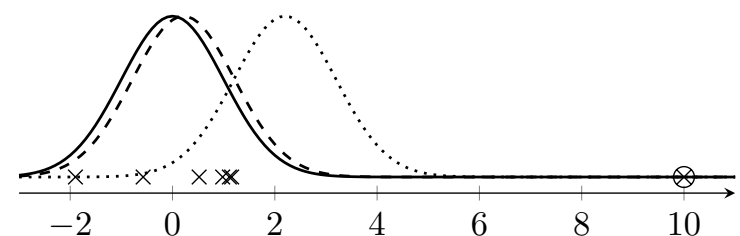

Fig. 2. Example of the drastic effect of outliers on estimated Gaussian distributions with known variance. We compare the estimates when no outliers are present in the data (dashed) and when there is one outlier at $x=10$ (dotted) with the true distribution of the data (solid).

Then, measurements of the output and, in some applications, of the input, are used to recover the posterior mean estimates of $w$ and $g$. Eventual hyperparameters are estimated from the marginal likelihood using an expectation-maximization algorithm [5] based on the empirical Bayes approach [6].

In the UI model, the measurement noise is modeled as a Gaussian random variable; however it is well known that the estimates obtained with quadratic penalization-that is, with Gaussian noise models-will be poor when there are outliers in the data [7], [8]. The problem is that the Gaussian distribution assigns very low probability to outcomes that are far away from the mean value; therefore, estimates of the distribution from data will be very affected by even a small number of outlier values. We illustrate this effect on a simple example. Suppose that we want to estimate the mean of a Gaussian distribution, with known variance, from a set of 6 measurements; the maximum-likelihood estimate of the mean is given by the sample average of the points:

$$
\hat{\mu}=\frac{1}{N} \sum_{i=1}^{N} x_{i} .
$$

Because all the observations have the same weight, they contribute in the same amount to the estimate of the mean. If we add an outlier (for instance, a sample 10 standard deviations away from the mean), the estimate (1) will shift by a very large amount (see Figure 2).

The problem of outlier-robust estimation is well studied and many techniques for robust regression have been proposed [9]-[11]. There have been outlier-robust approaches proposed also in system identification, often relying on noise models with heavy tails. For instance Student- $t$ distributions [12] or Laplace distributions [13]. The problem has been studied using time domain [14], [15], frequency domain [16], and 
subspace approaches [17]. Bayesian approaches for impulseresponse estimation in the presence of outliers have also been proposed [7], [8].

The contributions of this publication are as follows:

- By extending the UI model and estimation algorithm to handle data with outliers, we propose a modeling framework that is able to estimate a wide class of dynamic systems from data with outliers: with the proposed method we are able to identify nonparametric FIR models, Hammerstein models, cascaded linear systems, and to solve certain blind and errors-in-variables estimation problems, among others (see [1] for details).

- We validate the results in two simulation experiments: in the first, we consider nonparametric impulse response estimation and we show that the proposed method has a performance that is comparable to another state-ofthe-art Bayesian approach [8] (although it has a higher computational cost); in the second, we consider nonparametric Hammerstein model estimation and we show that, while classical UI models fail, the proposed robust UI framework has good performance also in presence of severe outliers in the data.

The rest of the paper is organized as follows. In Section II, we give a summary of the UI framework. In Section III, we present an outlier-robust extension of UI model. In Section IV, we present an iterative algorithm based on Markov chain Monte Carlo to estimate the hyperparameters of the model. In Section V, we present two simulation examples. In Section VI, we provide some conclusions and an outlook on future works. In the Appendix, we present proofs of the main results.

\section{SUMMARY OF THE UI FRAMEWORK}

In the UI model, we consider a stable linear time-invariant system represented by the $n \times 1$ vector of its impulse response samples $g$. If let $w$ denote the $N \times 1$ vector of samples of an input signal, we can write the $N \times 1$ vector of the output of the system (from zero initial conditions) as the noise-corrupted discrete convolution

$$
y=W g+\varepsilon,
$$

where $W$ is the $N \times n$ Toeplitz matrix of the input samples:

$$
[W]_{i, j}= \begin{cases}w_{i-j+1} & \text { if } 0<i-j+1<N, \\ 0 & \text { otherwise }\end{cases}
$$

and where $\varepsilon$ is an $N \times 1$ vector of noise samples. By linearity,

$$
W g=G w,
$$

where $G$ is the $N \times N$ lower-triangular Toeplitz matrix of the impulse response (padded with zeros).

To encode the knowledge we might have about the input signal and about the linear system, we use Gaussian-process models - that is, we model the vectors $g$ and $w$ as multivariate Gaussian vectors,

$$
g \sim \mathcal{N}\left(g ; 0, K_{\theta}\right), \quad w \sim \mathcal{N}\left(w ; 0, H_{\theta}\right)
$$

where the covariance matrices $K_{\theta}$ and $H_{\theta}$ can be used to encode prior information ${ }^{1}$. To allow for flexible models, we let the covariance matrices depend on the vector of hyperparameters $\theta$.

Together with the priors (4), we can define the likelihood function using (2) and assuming that the noise samples $\varepsilon_{k}$ are independent Gaussian random variables with precision (inverse variance) $\lambda$. Hence, the measurements have the density

$$
y \mid g, w, \lambda \sim \mathcal{N}\left(y ; W g, \Lambda^{-1}\right),
$$

where $\Lambda=\lambda I$ is the diagonal matrix of the noise precisions.

The UI model is defined by (4) and (5). In Figure 3 (left), we present the Bayesian network of the model in which we see that the $N$ output samples $\left\{y_{k}\right\}_{k=1}^{N}$ depend on the random variables $g$ and $w$ (whose densities act as priors).

We estimate the model, using an empirical Bayes approach [6], with the posterior means

$$
\begin{gathered}
\hat{g}:=\int g \mathrm{p}(g, w \mid y, \hat{\theta}, \hat{\lambda}) \mathrm{d} g \mathrm{~d} w, \\
\hat{w}:=\int w \mathrm{p}(g, w \mid y, \hat{\theta}, \hat{\lambda}) \mathrm{d} g \mathrm{~d} w,
\end{gathered}
$$

where the hyperparameters and the noise precision are estimated from the marginal likelihood:

$$
\hat{\theta}, \hat{\lambda}=\arg \max _{\theta, \lambda} \mathrm{p}(y \mid \theta, \lambda) \text {. }
$$

In the UI model, the marginal likelihood is intractable because it depends on the convolution of the Gaussian processes $g$ and $w$ which does not have a closed-form expression. To solve this issue, we interpret (7) as a maximum-likelihood problem with latent variables, according to

$$
\hat{\theta}, \hat{\lambda}=\arg \max _{\theta, \lambda} \int \mathrm{p}(y, g, w \mid \lambda, \theta) \mathrm{d} g \mathrm{~d} w,
$$

and use the EM method [5] to iteratively compute

1) $Q\left(\theta, \lambda ; \theta^{(j)}, \lambda^{(j)}\right):=$

$\int \log \mathrm{p}(y, g, w \mid \lambda, \theta) \mathrm{p}\left(g, w \mid y, \theta^{(j)}, \lambda^{(j)}\right) \mathrm{d} g \mathrm{~d} w$

2) $\theta^{(j+1)}, \lambda^{(j+1)}=\arg \max _{\theta, \lambda} Q\left(\theta, \lambda ; \theta^{(j)}, \lambda^{(j)}\right)$.

Under some technical conditions, $\theta^{(j)}$ and $\lambda^{(j)}$ will converge to a local solution of (8) [18].

Although we have circumvented the need to compute the marginal likelihood, the integrals in (6) and (9) are still intractable. Therefore, we approximate them by making a particle approximation of the joint posterior distribution,

$$
\mathrm{p}(g, w \mid y, \hat{\theta}, \hat{\lambda}) \approx \frac{1}{M} \sum_{i=1}^{M} \delta\left(g-\bar{g}^{(i)}\right) \delta\left(w-\bar{w}^{(i)}\right),
$$

where $\left\{\bar{g}^{(i)}\right\}$ and $\left\{\bar{w}^{(i)}\right\}$ are samples drawn from the posterior distribution $\mathrm{p}(g, w \mid y, \hat{\theta}, \hat{\lambda})$ using Gibbs sampling, which is an iterative method that samples from a joint distribution by in turn sampling each random variable conditioned on all the other variables [19]. Thus, to create the particle approximation (10), we can

\footnotetext{
${ }^{1}$ Because of space restrictions, we present only the case of zero-mean models; however, all the results remain valid in the more general case. (See [1] for details.)
} 
1) sample $g^{(i+1)} \mid y, w^{(i)}, \hat{\lambda}, \hat{\theta}$

2) sample $w^{(i+1)} \mid y, g^{(i+1)}, \hat{\lambda}, \hat{\theta}$,

for a large number of iterations (for a fixed value of the hyperparameters). This procedure creates a Markov chain of samples whose stationary distribution is the distribution we want to approximate. In practice, the initial samples have to be discarded because the Markov chain is not well mixed and is still far from the stationary distribution.

Because of (3), the conditional distributions of $g$ and $w$ are Gaussian distributions [1, Section 4.1]:

$$
\begin{aligned}
& g \mid y, w, \lambda, \theta \sim \mathcal{N}\left(g ; m_{g}, P_{g}\right), \\
& w \mid y, g, \lambda, \theta \sim \mathcal{N}\left(w ; m_{w}, P_{w}\right),
\end{aligned}
$$

with covariance matrices and means given by,

$$
\begin{aligned}
& P_{g}=\left(W^{T} \Lambda W+K_{\theta}^{-1}\right)^{-1}, \quad m_{g}=P_{g} W^{T} \Lambda y, \\
& P_{w}=\left(G^{T} \Lambda G+H_{\theta}^{-1}\right)^{-1}, \quad m_{w}=P_{w} G^{T} \Lambda y .
\end{aligned}
$$

Therefore, it is easy to sample from the joint posterior distribution with the Gibbs sampler and approximate the integral in (9) with a sample average over the particles,

$$
Q\left(\theta, \lambda ; \theta^{(j)}, \lambda^{(j)}\right) \approx \frac{1}{M} \sum_{i=1}^{M} \log \mathrm{p}\left(y, \bar{g}^{(i, j)}, \bar{w}^{(i, j)} \mid \theta, \lambda\right),
$$

where $\bar{g}^{(i, j)}$ and $\bar{w}^{(i, j)}$ are samples drawn, at the $j$ th iteration, from $\mathrm{p}\left(g, w \mid y, \hat{\lambda}^{(j)}, \hat{\theta}^{(j)}\right)$. This method of approximating (9) is known as Monte-Carlo EM (MCEM) [20]. Thanks to the structure of the problem, we have that the update of the hyperparameters at the $j$ th iteration depends only on certain moments computed from the Gibbs sampler: let

$$
\begin{aligned}
S_{g}^{(j)} & =\frac{1}{M} \sum_{i=1}^{M} \bar{g}^{(i, j)} \bar{g}^{(i, j) T}, \quad S_{w}^{(j)}=\frac{1}{M} \sum_{i=1}^{M} \bar{w}^{(i, j)} \bar{w}^{(i, j) T}, \\
v^{(j)} & =\frac{1}{M} \sum_{i=1}^{M}\left\|y-\bar{W}^{(i, j)} \bar{g}^{(i, j)}\right\|^{2},
\end{aligned}
$$

where $\bar{W}^{(i, j)}$ is the $N \times n$ Toeplitz matrix of the sample $\bar{w}^{(i, j)}$; then, we have the following result.

Theorem 1: At the $j$ th iteration of the MCEM method, the update of the hyperparameters is given by

$$
\begin{aligned}
& \theta^{(j+1)}=\arg \min _{\theta} \operatorname{Tr}\left\{K_{\theta}^{-1} S_{g}^{(j)}+H_{\theta}^{-1} S_{w}^{(j)}\right\}+\log \operatorname{det}\left(K_{\theta} H_{\theta}\right), \\
& \lambda^{(j+1)}=N / v^{(j)} .
\end{aligned}
$$

Proof: See [1, Section 5.2].

Once the iterations of the MCEM method have converged, we can run another Gibbs sampler to find $\bar{g}^{(i)}$ and $\bar{w}^{(i)}$ and approximate (6) with

$$
\hat{g} \approx \frac{1}{M} \sum_{i=1}^{M} \bar{g}^{(i)}, \quad \hat{w} \approx \frac{1}{M} \sum_{i=1}^{M} \bar{w}^{(i)} .
$$

As we argued in the introduction, the assumption in (5) may lead to a lack of robustness in cases where the noise in the measurements is not Gaussian. In the next section, we present an extension of the UI framework to handle outlier-robust estimation whereby one sampling step is added to the Gibbs sampler and the update (12) is replaced with a maximization with respect to two additional hyperparameters.
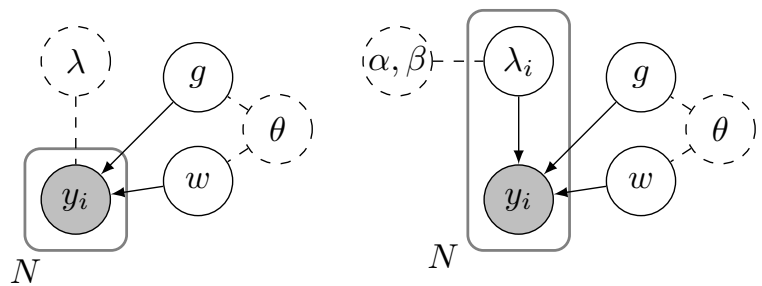

Fig. 3. Bayesian networks of models considered in this paper. Solid circles represent stochastic variables; dashed circles represent deterministic parameters; arrows represent dependency relationships; full circles represent observed variables. The plate represents the $N$ different samples. The network on the left represents the UI model of [1]; the network on the right represents the outlier-robust UI model.

\section{THE OUTLIER-ROBUST UI FRAMEWORK}

To allow for noise models with heavy tails, we use a compound Gaussian model where the precision of each noise sample is a random variable with a Gamma density with shape parameter $\alpha$ and scale parameter $\beta$ :

$$
\lambda_{k} \sim \mathrm{Ga}\left(\lambda_{k} ; \alpha, \beta\right) \propto \lambda_{k}^{\alpha-1} \exp \left\{-\lambda_{k} / \beta\right\},
$$

This implies that the each noise sample has a scaled Student- $t$ distribution with $2 \alpha$ degrees of freedom and shape $(\alpha \beta)^{-1}$ :

$$
\varepsilon_{k} \sim \operatorname{St}\left(\varepsilon_{k} ; 2 \alpha,(\alpha \beta)^{-1}\right) \propto\left\{1+\beta x^{2} / 2\right\}^{-\alpha-1 / 2} .
$$

If we fix $\alpha=1 / 2$, we get a Cauchy model for the noise.

Thus, the outlier-robust UI model consists of (4), (5), and (14). In Figure 3 (right), we present the Bayesian network of the outlier-robust UI model; note that the noise precision is now a random variable with a prior distribution.

The hyperparameters to be estimated are the kernel hyperparameters $\theta$ and the parameters $\alpha$ and $\beta$ of the precision distribution. These are estimated from the marginal distribution of $y$ after integrating out $\lambda$ (in addition to $g$ and $w$ ). In the next section, we present an iterative estimation algorithm based on MCEM to estimate the outlier-robust UI model.

\section{ITERATIVE ESTIMATION ALGORITHM}

As was the case for the UI model, in the outlier-robust UI model we estimate the hyperparameters from the marginal likelihood using the MCEM method. To this end, we define the $Q$ function, at the $j$ th iteration, as the expectation of the complete-data likelihood with respect to the particle approximation of the distribution of $g, w$, and $\lambda$, given $y$ :

$$
\begin{aligned}
& Q\left(\theta, \alpha, \beta ; \theta^{(j)}, \alpha^{(j)}, \beta^{(j)}\right) \approx \\
& \frac{1}{M} \sum_{i=1}^{m} \log \mathrm{p}\left(y, \bar{g}^{(i, j)}, \bar{w}^{(i, j)}, \bar{\lambda}^{(i, j)} \mid \theta, \alpha, \beta\right),
\end{aligned}
$$

where $\bar{g}^{(i, j)}, \bar{w}^{(i, j)}$, and $\bar{\lambda}^{(i, j)}$ are samples from the joint posterior of the latent variables given the data.

The Bayesian network in Figure 3 (right) suggests that we can again use the Gibbs sampling algorithm to sample the latent variables in the outlier-robust UI model. To this end, we have the following result. 
Lemma 2: Consider the outlier-robust UI model given by (4), (5), (14), then

$$
\begin{aligned}
g \mid y, w, \lambda, \theta & \sim \mathcal{N}\left(g ; m_{g}, P_{g}\right), \\
w \mid y, w, \lambda, \theta & \sim \mathcal{N}\left(w ; m_{w}, P_{w}\right), \\
\lambda_{k} \mid y, g, w, \alpha, \beta & \sim \operatorname{Ga}\left(\lambda_{k} ; \alpha_{k}, \beta_{k}\right), \quad k=1, \ldots, N .
\end{aligned}
$$

where $m_{g}, P_{g}, m_{w}$, and $P_{w}$ are given in (11) and where

$$
\alpha_{k}=\alpha+\frac{1}{2}, \quad \frac{1}{\beta_{k}}=\frac{1}{\beta}+\frac{1}{2}\left(y_{k}-w_{k}^{T} g\right)^{2},
$$

where $w_{k}^{T}$ is the $k$ th row of $W$.

Thanks to Lemma 2, we can easily sample the posterior distribution of the latent variables with standard tools. At the $j$ th iteration of the MCEM method, we compute the sample second moments $S_{g}^{(j)}$ and $S_{w}^{(j)}$, as well as the arithmetic and geometric means of the sampled precisions,

$$
\lambda_{\mathrm{A}}^{(j)}=\frac{1}{N M} \sum_{k=1}^{N} \sum_{i=1}^{M} \lambda_{k}^{(i, j)}, \quad \lambda_{\mathrm{G}}^{(j)}=\left(\prod_{k=1}^{N} \prod_{i=1}^{M} \lambda_{k}^{(i, j)}\right)^{\frac{1}{M N}} .
$$

Then, we have the following result.

Theorem 3: At the $j$ th iteration of the MCEM method, the update of the hyperparameters for the outlier-robust UI model is given by

$$
\begin{aligned}
& \theta^{(j+1)}=\arg \min _{\theta} \operatorname{Tr}\left\{K_{\theta}^{-1} S_{g}^{(j)}+H_{\theta}^{-1} S_{w}^{(j)}\right\}+\log \operatorname{det}\left(K_{\theta} H_{\theta}\right), \\
& \alpha^{(j+1)}=\arg \min _{\alpha} \alpha \log \left(\lambda_{\mathrm{A}}^{(j)} / \lambda_{\mathrm{G}}^{(j)}\right)+\log \Gamma(\alpha)+\alpha \\
& \beta^{(j+1)}=\lambda_{\mathrm{A}}^{(j)} / \alpha^{(j+1)} .
\end{aligned}
$$

where $\Gamma(\cdot)$ is the Gamma function.

Once the iterations of the MCEM method have converged, we can run another Gibbs sampler, discard the samples drawn for $\lambda$, and find the approximations of (6) as shown in (13).

Note that, if we want to use a Cauchy model for the noise, the update of the hyperparameters becomes $\alpha^{(j+1)}=\alpha^{(j)}=$ $1 / 2$ and $\beta^{(j+1)}=2 \lambda_{\mathrm{A}}^{(j)}$.

Remark 4: In the general case, original UI framework requires the sampling of an $N$-dimensional vector at each iteration. Using Choleski factorization, this has an asymptotic time complexity of $O\left(N^{3}+N^{2} M\right)$ per iteration. In the robust case, the computational complexity is not increased as the additional steps required (sampling $\lambda$ and solving for $\alpha$ and $\beta$ ) have lower asymptotic complexity than the sampling step. Of course, specific instances of UI models can have lower computational complexity (see, for instance, Section V-A).

\section{Simulations}

In this section, we present two simulated experiments in which we use the proposed method to estimate nonparametric FIR and Hammerstein systems. In the first experiment, we show that the proposed method has a performance that is close to the performance of state-of-the-art methods for the estimation of nonparametric FIR models. In the second experiment, we tackle the problem of nonparametric Hammerstein estimation and we show that, while standard UI methods fail in presence of outliers, the proposed outlier-robust UI method is able to effectively estimate the system.

\section{A. Estimation of linear systems}

In this section, we estimate the impulse responses of the first 100 systems from the S1D1 dataset [3]. We extract the impulse responses of the systems in the dataset and we simulate $N=200$ samples of the outputs to Gaussian white-noise inputs $u$ with unitary variance. We consider two experiments: in the first, we add Gaussian white measurement noise with variance $10 \%$ of the noiseless output of the systems; in the second, in addition to the noise, we add outliers by selecting (without replacement) $N_{\text {out }}=20$ samples of the output and corrupting them with Gaussian noise with variance 100 times the corresponding noiseless output variance.

In the simulation, we compare the following methods:

SS A nonparametric kernel-based approach [4]. We model the impulse response of the system with a first-order stable spline kernel [21],

$$
\left[K_{\theta}\right]_{i, j}=\theta_{1} \theta_{2}^{\max (i, j)},
$$

and we estimate the impulse response as the posterior mean given the data. The hyperparameters are estimated from the marginal likelihood. Note that this method uses a Gaussian noise model and is therefore not robust to outliers.

T-UI The proposed outlier-robust approach with Student$t$ model for the noise. The input is known exactly so we model $w \sim \mathcal{N}(w ; u, 0)$ - that is, we never sample $w$ and set it always equal to the input $u$ which lowers the time complexity per iteration to $O\left(n^{3}+n^{2} M\right)$ (see Remark 4). The impulse response is modeled with (17). At each iteration of the MCEM method, we discard 50 samples for burn-in and simulate $M=100$ samples. The iterations are stopped with a relative tolerance of $10^{-3}$. The final impulse response is estimated with 1000 samples (after 200 samples of burn-in).

C-UI The proposed approach with the Cauchy model for the noise. The same method as T-UI with $\alpha=1 / 2$.

EM-S An outlier-robust EM kernel-based method [8]. It models the impulse response of the linear system with (17) and the noise as independent Student- $t$ random variables. The noise variances and the hyperparameters of the stable-spline model are estimated from the marginal likelihood using the EM method. The degrees of freedom of the Student- $t$ distribution are selected with a model-selection technique. This method has an asymptotic computational complexity of $O\left(n^{2.373}\right)$ per iteration (a matrix inversion).

EM-L The same kernel-based method as EM-S, with the Laplace model for the noise [8].

To evaluate the performance of the methods, we use the normalized fit score

$$
\operatorname{Fit}(\hat{g})=1-\frac{\left\|g_{0}-\hat{g}\right\|_{2}}{\left\|g_{0}-\tilde{g}_{0}\right\|_{2}}
$$

where $g_{0}$ is the true value of the impulse response and $\tilde{g}_{0}$ is the sample mean of $g_{0}$. 


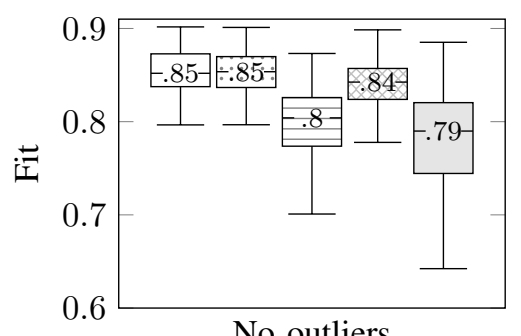

No outliers

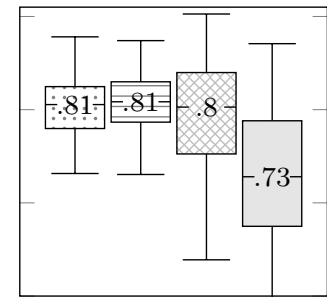

$10 \%$ outliers $\square$ SS $\cdots \mathrm{T}$-UI $\rightleftarrows \mathrm{C}$-UI $\bowtie \mathrm{EM}-\mathrm{S} \square \mathrm{EM}-\mathrm{L}$

Fig. 4. Boxplots of the fits of the estimate of the impulse responses of the first 100 systems in the S1D1 dataset. Left: no outliers are present in the data. Right: $10 \%$ of the output samples are corrupted with outliers with variance 100 times the noiseless output variance (we omit the plot for SS because it has negative fit).

The results of this simulation are presented in Figure 4. In the figure we see the boxplots of the fits of the estimates over the 100 systems in the dataset. From the simulation, it appears that the proposed approach, when applied to the problem of estimation of nonparametric FIR models, compares favorably to state of the art kernel-based methods both in cases without outliers and when the data are corrupted by outliers.

\section{B. Estimation of Hammerstein systems}

In this section, we present two experiments. In each experiment we estimate the impulse responses and static nonlinearities of 150 Hammerstein systems from data with outliers. All Hammerstein systems are generated with the following procedure:

1) The linear block is a discrete-time transfer function of random order $m$ uniformly drawn in the set $\{3,4,5\}$. The static nonlinearity is a polynomial of random order $p$ uniformly drawn in the set $\{5,6, \ldots, 10\}$.

2) We generate $m$ poles (in complex-conjugate pairs) drawing magnitudes uniformly in [0.4, 0.8] and $m-1$ zeros (in complex-conjugate pairs) drawing magnitudes uniformly in $[0,0.9]$. All the phases are sampled uniformly in $[0, \pi / 3]$. We generate the static nonlinearity as a combination of the first $p$ Legendre polynomials, with coefficients drawn uniformly in $[-1,1]$.

3) We generate a uniform white noise input in $[-1,1]$ and we collect $N=300$ samples of the output, from zero initial conditions, with white measurement noise with variance $10 \%$ of the variance of the noiseless output.

To represent the outliers in the data, we randomly select, without replacement, $N_{\text {out }}$ samples of the output and we corrupt them with Gaussian noise with variance that is a multiple of the noiseless output variance.

In the simulations, we compare the two estimators

G-UI The method presented in [1] for the estimation of Hammerstein systems. It uses the first-order stable spline kernel (17) for the impulse response and a squared-exponential kernel for the nonlinearity:

$$
\left[H_{\theta}\right]_{i, j}=\exp \left\{-\theta_{3}\left(u_{i}-u_{j}\right)^{2}\right\} .
$$
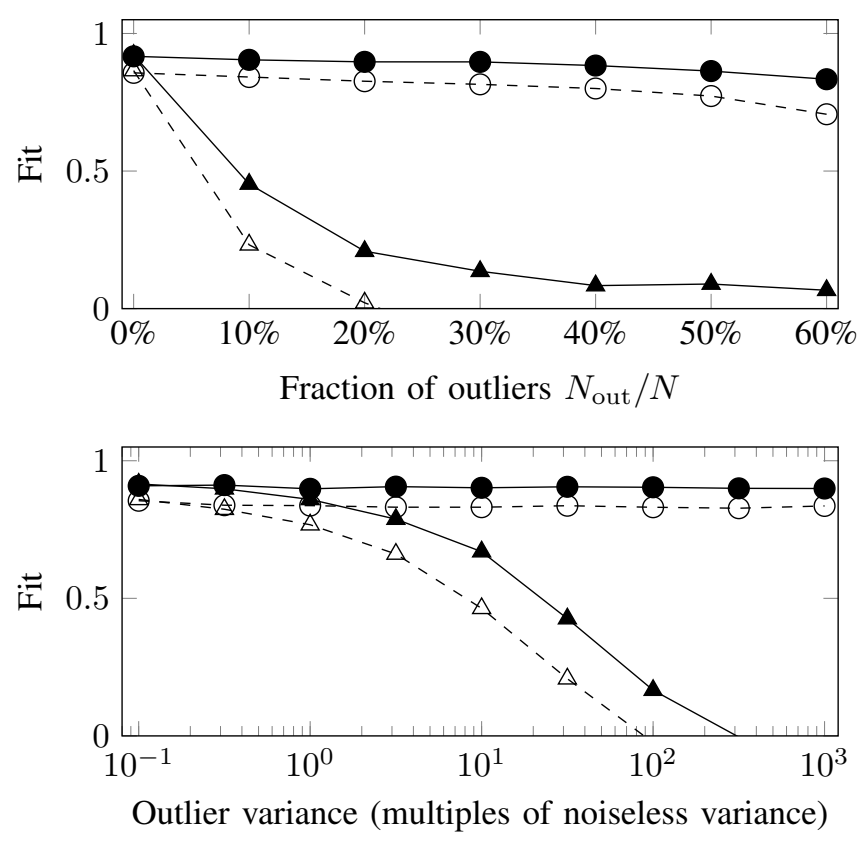

$$
-\hat{g}_{\mathrm{T}-\mathrm{UI}}-\ominus-\hat{f}_{\mathrm{T}-\mathrm{UI}}-\hat{g}_{\mathrm{G}-\mathrm{UI}}-\Delta-\hat{f}_{\mathrm{G}-\mathrm{UI}}
$$

Fig. 5. Median fit of the methods over the 150 Hammerstein systems in the dataset. Circles refer to the proposed outlier-robust estimator; triangles to the UI estimator in [1]. Solid plots refer to estimates of the impulse response; dashed plots to estimates of the static nonlinearity. Top: experiment with different fractions of outliers. Bottom: experiment with different values of the variance of the outliers.

The parameters are estimated as presented in Section II. At each iteration of the MCEM method we discard 100 samples from the Gibbs sampler for burnin and then draw $M=200$ samples. The iterations are stopped once the relative change in the parameters is below $10^{-3}$. Then, $M=1000$ samples are used to compute the estimates (6).

T-UI The proposed outlier-robust estimation method with $t$-distributed noise. It uses the same kernels and the same parameters for the MCEM method as G-UI.

Using the methods, we compute the estimates $\hat{g}_{\mathrm{G}-\mathrm{UI}}$ and $\hat{g}_{\text {T-UI }}$ of the first $n=45$ samples of the impulse response of the linear block, and the estimates $\hat{f}_{\mathrm{G}-\mathrm{UI}}$ and $\hat{f}_{\mathrm{T}-\mathrm{UI}}$ of the static nonlinearity evaluated at a uniform grid of 100 values in $[-1,1]$. We evaluate the accuracy of the estimates of the impulse response and of the static nonlinearity with normalized fit measures similar to (18).

In the first experiment, we consider different values of $N_{\text {out }}$ between 0 (no outliers) and 180 (60\% of outliers in the data). The results are presented in Figure 5 (top). The figure shows the fit of the models estimated with the T-UI method (circles) and the G-UI method (triangles). From the figure, it is clear that the outlier-robust method has better performance both in reconstructing the impulse response (solid line) and static nonlinearity (dashed line) compared to the G-UI method which has increasingly worse performance for higher fractions of outliers. 
In the second experiment, we consider $N_{\text {out }}=45$ ( $15 \%$ of outliers in the data) and we vary the variance of the outliers between $1 / 10$ and 1000 times the noiseless output variance. The results are presented in Figure 5 (bottom). From the figure, we can draw the same conclusions as we did in the first experiment: for increasing outlier variance, the Gaussiannoise assumption in G-UI impacts the performance negatively whereas the proposed outlier-robust UI method is insensitive even to very high outlier variances.

\section{CONCLUSIONS AND FUTURE WORKS}

In this paper, we have presented an extension of the UI model suitable for estimation when the data are corrupted by outliers. The UI model consists of a linear system subject to a partially unknown input. The impulse response of the linear system and the unknown input were modeled as Gaussian processes. In the proposed robust extension, we used a noise model with heavy tails to model outliers in the measurements. We have proposed a Gibbs sampling algorithm to estimate the model which consists in a simple extension of the original UI algorithm. We have validated the approach on a standard dataset of linear systems, where we have shown that the proposed robust method has a performance that is comparable to state-of-the-art algorithms, and on a dataset of Hammerstein systems where we have shown that the proposed extension compensates for the lack of robustness of the original UI model with respect to outliers.

In its current formulation, the UI framework requires sampling a vector that has the same dimension as the data at each iteration of the Gibbs sampler; to reduce this complexity, it is possible to use variational Bayes or sparse Gaussian processes to simplify the sampling steps or stochastic approximation expectation-maximization to reduce the number of steps required. These developments will be explored in future papers.

\section{APPENDIX}

Proof of Lemma 2: The proof of the normality of $g \mid y, w, \lambda, \theta$ and $w \mid y, w, \lambda, \theta$ follows the same arguments as the proof in [1, Section 5.2]. Regarding $\lambda_{k} \mid y, g, w, \alpha, \beta$,

$$
\begin{aligned}
\mathrm{p}\left(\lambda_{k} \mid y, g, w, \alpha, \beta\right) & \propto \mathrm{p}\left(\lambda_{k} \mid \alpha, \beta\right) \mathrm{p}\left(y_{k} \mid g, w, \lambda\right) \\
& \propto \lambda_{k}^{a-\frac{1}{2}} \exp \left\{-\lambda_{k}\left(\frac{1}{\beta}+\frac{1}{2}\left(y_{k}-w_{k}^{T} g\right)^{2}\right)\right\} .
\end{aligned}
$$

So, $\lambda_{k} \mid y, g, w, \alpha, \beta$ is Gamma distributed with the indicated parameters.

Proof of Theorem 3: Consider the likelihood of the robust UI model:

$$
\begin{aligned}
& \log \mathrm{p}(y, g, w, \lambda \mid \theta, \alpha, \beta)=\log \mathrm{p}(g \mid \theta) \mathrm{p}(w \mid \theta) \mathrm{p}(y \mid g, w, \lambda) \mathrm{p}(\lambda \mid \alpha, \beta) \\
& =-\frac{1}{2} g^{T} K_{\theta}^{-1} g-\frac{1}{2} \log \operatorname{det} K_{\theta}-\frac{1}{2} w^{T} H_{\theta}^{-1} w-\frac{1}{2} \log \operatorname{det} H_{\theta} \\
& +\sum_{k=1}^{N}\left\{-\alpha \log \beta-\log \Gamma(\alpha)+(\alpha-1) \log \lambda_{k}-\frac{\lambda_{k}}{\beta}\right\}+\text { const. }
\end{aligned}
$$

Consider now the particle approximation (15). Using the fact that $b^{T} A b=\operatorname{Tr}\left\{A b b^{T}\right\}$, we find that

$$
Q\left(\theta, \alpha, \beta ; \theta^{(j)}, \alpha^{(j)}, \beta^{(j)}\right) \approx-\frac{1}{2} \operatorname{Tr}\left\{K_{\theta}^{-1} S_{g}^{(j)}+H_{\theta}^{-1} S_{w}^{(j)}\right\}
$$

$$
\begin{aligned}
& -\frac{1}{2} \log \operatorname{det}\left(K_{\theta} H_{\theta}\right)-N \alpha \log \beta-N \log \Gamma(\alpha) \\
& +(\alpha-1) \frac{\sum_{i=1}^{M} \sum_{k=1}^{N} \log \lambda_{k}^{(i, j)}}{\beta}-\frac{\sum_{i=1}^{M} \sum_{k=1}^{N} \lambda_{k}^{(i, j)}}{\beta}
\end{aligned}
$$

where we have dropped all terms independent of the hyperparameters. Maximizing with respect to $\theta$, we find the first update. Then, using the definitions in (16), we have that

$$
\frac{\partial}{\partial \beta} Q\left(\theta, \alpha, \beta ; \theta^{(j)}, \alpha^{(j)}, \beta^{(j)}\right)=-\frac{N \alpha}{\beta}-\frac{\lambda_{\mathrm{A}}^{(j)}}{\beta^{2}},
$$

which gives us the update for $\beta$. Plugging the optimal $\beta$ back into the cost function, we get the update of $\alpha$

\section{REFERENCES}

[1] R. S. Risuleo, System identification with input uncertainties: an EM kernel-based approach. KTH - Royal Institute of Technology, 2016.

[2] C. E. Rasmussen and C. K. Williams, Gaussian processes for machine learning. the MIT Press, 2006.

[3] T. Chen, H. Ohlsson, and L. Ljung, "On the estimation of transfer functions, regularizations and Gaussian processes-Revisited," Automatica, vol. 48, no. 8, pp. 1525-1535, 2012.

[4] G. Pillonetto, F. Dinuzzo, T. Chen, G. De Nicolao, and L. Ljung, "Kernel methods in system identification, machine learning and function estimation: A survey," Automatica, vol. 50, no. 3, pp. 657-682, 2014.

[5] A. P. Dempster, N. M. Laird, and D. B. Rubin, "Maximum likelihood from incomplete data via the EM algorithm," J. R. Stat. Soc. Ser. B (Methodol.), pp. 1-38, 1977.

[6] J. S. Maritz and T. Lwin, Empirical Bayes methods. Chapman and Hall London, 1989.

[7] G. Bottegal, H. Hjalmarsson, A. Y. Aravkin, and G. Pillonetto, "Outlier robust kernel-based system identification using $\ell_{1}$-Laplace techniques," in Proc. IEEE Conf. Decis. Control (CDC), 2015.

[8] G. Bottegal, A. Y. Aravkin, H. Hjalmarsson, and G. Pillonetto, "Robust EM kernel-based methods for linear system identification," Automatica, vol. 67 , pp. 114-126, 2016.

[9] P. J. Huber, "Robust estimation of a location parameter," Ann. Math. Statist., pp. 73-101, 1964.

[10] P. Rousseeuw and V. Yohai, "Robust regression by means of Sestimators," in Robust and nonlinear time series analysis, pp. 256-272, Springer, 1984.

[11] P. J. Huber, "Robust statistics," in International Encyclopedia of Statistical Science, pp. 1248-1251, Springer, 2011.

[12] M. E. Tipping and N. D. Lawrence, "Variational inference for studentt models: Robust bayesian interpolation and generalised component analysis," Neurocomputing, vol. 69, no. 1-3, pp. 123-141, 2005.

[13] A. Y. Aravkin, B. M. Bell, J. V. Burke, and G. Pillonetto, "An $\ell_{1}$-Laplace robust Kalman smoother," IEEE Trans. Autom. Control, vol. 56, no. 12, pp. 2898-2911, 2011.

[14] J. L. Rojo-Álvarez, M. Martínez-Ramón, M. de Prado-Cumplido, A. Artés-Rodríguez, and A. R. Figueiras-Vidal, "Support vector method for robust ARMA system identification," IEEE Trans. Signal Process., vol. 52, no. 1, pp. 155-164, 2004.

[15] J. Dahlin, F. Lindsten, T. B. Schön, and A. Wills, "Hierarchical Bayesian ARX models for robust inference," IFAC Proc. Vol., vol. 45, no. 16, pp. 131-136, 2012.

[16] E. Goberdhansigh, L. Wang, and W. R. Cluett, "Robust frequency domain identification," Chem. Eng. Sci., vol. 47, no. 8, pp. 1989-1999, 1992.

[17] D. Sadigh, H. Ohlsson, S. S. Sastry, and S. A. Seshia, "Robust subspace system identification via weighted nuclear norm optimization," IFAC Proceedings Volumes, vol. 47, no. 3, pp. 9510-9515, 2014.

[18] C. F. J. Wu, "On the convergence properties of the EM algorithm," Ann. Statist., vol. 11, no. 1, pp. 95-103, 1983.

[19] S. Geman and D. Geman, "Stochastic relaxation, Gibbs distributions, and the Bayesian restoration of images," IEEE Trans. Pattern Anal. Mach. Intell., no. 6, pp. 721-741, 1984

[20] G. C. G. Wei and M. A. Tanner, "A Monte Carlo implementation of the EM algorithm and the poor man's data augmentation algorithms," $J$. Am. Stat. Assoc., vol. 85, no. 411, pp. 699-704, 1990.

[21] G. Pillonetto, A. Chiuso, and G. De Nicolao, "Prediction error identification of linear systems: a nonparametric gaussian regression approach," Automatica, vol. 47, no. 2, pp. 291-305, 2011. 